\title{
Intrinsic vs. spurious long-range memory in high-frequency records of environmental radioactivity
}

\author{
Critical re-assessment and application to indoor ${ }^{222} \mathbf{R n}$ \\ concentrations from Coimbra, Portugal
}

Reik V. Donner ${ }^{1, a}$, Stelios M. Potirakis ${ }^{2}$, Susana M. Barbosa ${ }^{3,4}$, José A.O. Matos ${ }^{5}$, Alcides J.S.C. Pereira ${ }^{6}$, and Luis J.P.F. Neves ${ }^{6}$

1 Research Domain IV - Transdisciplinary Concepts \& Methods, Potsdam Institute for Climate Impact Research, Telegrafenberg A31, 14473 Potsdam, Germany

2 Department of Electronics Engineering, Piraeus University of Applied Sciences, 250 Thivon \& P. Ralli, 12244 Aigaleo, Athens, Greece

3 Center for Information Systems and Computer Graphics, INESC TEC, Campus da FEUP, Rua Dr. Roberto Frias, 4200-464 Porto, Portugal

4 Instituto Dom Luiz, University of Lisbon, Campo Grande, Edificio C8, 1749-016 Lisbon, Portugal

${ }^{5}$ Faculty of Economics, University of Porto, Rua Dr. Roberto Frias, 4200-464 Porto, Portugal

${ }^{6}$ CEMUC - Department of Earth Sciences, University of Coimbra, 3030-790 Coimbra, Portugal

Abstract. The presence or absence of long-range correlations in the environmental radioactivity fluctuations has recently attracted considerable interest. Among a multiplicity of practically relevant applications, identifying and disentangling the environmental factors controlling the variable concentrations of the radioactive noble gas radon is important for estimating its effect on human health and the efficiency of possible measures for reducing the corresponding exposition.

In this work, we present a critical re-assessment of a multiplicity of complementary methods that have been previously applied for evaluating the presence of long-range correlations and fractal scaling in environmental radon variations with a particular focus on the specific properties of the underlying time series. As an illustrative case study, we subsequently re-analyze two high-frequency records of indoor radon concentrations from Coimbra, Portugal, each of which spans several weeks of continuous measurements at a high temporal resolution of five minutes.

Our results reveal that at the study site, radon concentrations exhibit complex multi-scale dynamics with qualitatively different properties at different time-scales: (i) essentially white noise in the high-frequency part (up to time-scales of about one hour), (ii) spurious indications of a non-stationary, apparently long-range correlated process (at time scales between some hours and one day) arising from marked periodic 
components, and (iii) low-frequency variability indicating a true longrange dependent process. In the presence of such multi-scale variability, common estimators of long-range memory in time series are prone to fail if applied to the raw data without previous separation of time-scales with qualitatively different dynamics.

\section{Introduction}

Identifying and quantifying environmental factors controlling the fluctuations of natural radioactivity is one of the great open challenges in this field of research [1]. Among others, the concentration of the radioactive noble gas radon in soil, water and air has been considered as a potential tracer for a broad variety of geological and geophysical processes ranging from groundwater contamination [2] over preparatory stages of earthquakes [3-5] to atmospheric dynamics [6]. One of the most direct consequences are health hazards due to exhalation of the radioactive gas emitted from the ground in certain geological environments, which is known to be one of the major causes of lung cancer in many regions of the world [7]. All these examples underline that a better understanding of the dynamics of radon fluctuations is key to quantifying environmental risks in many areas.

It is widely accepted that the variability of radon concentrations can be affected by a multiplicity of mechanisms involving geophysical as well as climatological variables as potential controlling factors [1]. Identifying and disentangling these factors is, however, a highly context-specific and still largely unsolved problem. The main reasons for this are the typical properties of radon time series, which strongly restrict the applicability of most common techniques from linear as well as nonlinear time series analysis. Particularly problematic features typically found in recordings of radon fluctuations are:

multi-scale dynamics: In many situations, radon variations exhibit marked diurnal as well as annual cycles. These cycles could be related to either climatic (e.g., temperature, air pressure) or geophysical controls (tidal forces), and a clear attribution to either of both types of mechanisms has not yet been achieved. In addition, radon time series often exhibit complex variability patterns on other time-scales as well, ranging from hours to multi-day variability [8-10].

heteroskedasticity: This feature often arises associated with the emergence of periodic components, where the overall level of variability (i.e., the variance of fluctuations) depends on the mean amplitude of fluctuations [11,12]. For example, in many cases both the overall radon concentration and its temporal fluctuations are larger during day than night-time.

non-normality: The probability distribution function (PDF) of radon concentrations estimated from observational records is often not symmetric and, hence, cannot be appropriately approximated by a Gaussian distribution (which is taken as a common assumption in many classical time series analysis methods). There are various factors that may contribute to this behavior. First, one potential mechanism naturally leading to such a non-normality could be the combination of diurnal or seasonal cycles in mean and variance of radon concentrations $[12,13]$. Second, variables resulting from a multiplicity of independent random factors are expected to follow a log-normal distribution according to the central limit theorem, which could be another explanation for the observed non-normality of radon time series [14-17]. However, due to the complex dependence of radon emanation on the geological setting and the (unknown) history of (possibly mutually dependent) physical processes, a log-normal PDF does

a e-mail: reik.donner@pik-potsdam.de 
not necessarily provide a reasonable model assumption either [18]. Finally, we emphasize that ${ }^{222} \mathrm{Rn}$ concentrations are obtained from detections of ensembles of particles individually emitted by radioactive decay. Since the decay processes of individual atoms are mutually independent of each other, considering a Poisson process as a stochastic model for particle emission is a reasonable approximation, providing yet another explanation for the non-normality of radon time series.

non-stationarity: Beyond the complex interplay of variability patterns with different natural time-scales, the relative contribution of these scales also commonly varies with time, making the resulting radon recordings non-stationary. As a consequence, many traditional methods of linear time series analysis (such as correlation analysis or the closely related spectral analysis) are unable to properly capture these dynamical patterns and may thus cause misleading results.

non-linearity: Finally, it is notable that due to the multiplicity of potential external factors controlling radon concentrations, such time series commonly cannot be approximated by autonomous linear stochastic processes, but require the involvement of nonlinear terms and/or explicit external forces to statistically describe the observed dynamics [11].

Based on these generic features of observed radon time series, a critical discussion of possible strategies contributing to a statistical-dynamical process characterization of radon variations appears necessary. We emphasize that the aforementioned features are not unique to radon, but are also exhibited by other types of geophysical time series from a wide range of fields. Therefore, we believe that the considerations presented in this manuscript are also valid and relevant for a broad range of geoscientific problems beyond fluctuations of environmental radioactivity.

As a specific aspect, in the present work we focus on the problem of assessing the general type of correlations underlying radon time series. Under general conditions, there are two different types of correlations: short-range dependent time series, where the influence of previous values decays exponentially with time, and longrange dependent time series, where this decay rather follows a power-law $[19,20]$. While short-range dependent stochastic processes are often considered as benchmark models of "environmental noise", processes exhibiting long-range memory are equally wide-spread in Earth sciences. Moreover, one needs to distinguish persistent from anti-persistent behavior, i.e., cases where the subsequent increments of the process under study are positively and negatively correlated, respectively. For this purpose, one convenient way is estimating the Hurst exponent $H$ [21], which can be assessed using a multiplicity of techniques $[22-24,20]$. Specifically, $H>0.5$ for persistent and $H<0.5$ for anti-persistent behavior, whereas the case $H=0.5$ corresponds to the dynamics exhibited by classical Brownian motion (i.e., a process with uncorrelated increments).

In the context of the present work, we emphasize that identifying and quantitatively characterizing the possible presence of long-range memory (in combination with the associated persistence properties) in radon variability presents one of the first steps to constraining the underlying dynamical processes and their possible environmental drivers.

Over the last two decades, there have been various studies on the presence of long-range memory in radon time series obtained under very different conditions (see Tab. 1 for an overview of selected recent results). Notably, these studies have lead to inconclusive and partly even contradictory results, suggesting cases with persistent as well as anti-persistent dynamics without allowing for a clear discrimination between settings leading to one or the other type of dynamics. One main reason for this inconsistency could be the utilization of different methods of time series analysis in these recent studies, which are based on different assumptions on the inherent properties of the data under study. However, in many recent publications, an in- 
depth discussion of the latter assumptions and if they have been met in the case of the respective time series (particularly given the common observational features of radon time series mentioned above) has been missing.

This work provides a critical discussion of some methods for detecting long-range dependence that have been previously applied in the context of radon time series analysis. For this purpose, we initially provide a brief summary of selected approaches together with a short discussion of their intrinsic assumptions and practical limitations. In order to illustrate the performance of these methods, we re-examine some highfrequency records of indoor radon concentrations from Coimbra (Portugal), which have been previously studied [8] by some of the authors of this work with respect to periodicities (e.g., due to tidal forces) contained in these data and their possible physical constraints (e.g., a possible driving by different atmospheric variables such as air temperature and pressure), revealing a multi-scale structure of the observed variability pattern. We emphasize that a more detailed assessment of the relevant statistical and dynamical properties of such multi-scale dynamics is a challenging task by itself and may relate to phenomena such as multifractality, but also the necessity of proper time-scale decomposition of the data. Regarding the variability of radon concentrations, the two latter aspects have been addressed recently by various authors [39-43] for some specific geological settings.

This paper is organized as follows: In Section 2, we describe some methodological approaches for assessing long-range dependence and fractal scaling in radon time series. The data used for exemplifying the performance of these different approaches are described in Section 3. Section 4 describes the results obtained with the different methods and highlights their conceptual problems when applied to typical radon data. A possible strategy for solving of some of these problems based on time series decomposition is presented and further discussed in Section 5.

\section{Detecting long-range memory in observational data}

In this section, we provide an overview on some of the techniques that have been previously applied for studying long-range dependence in radon time series. The list is not fully exclusive, for example, we intentionally exclude the classical $\mathrm{R} / \mathrm{S}$ analysis [21] since it is known to exhibit rather problematic features in terms of bias and variance when being applied to, e.g., non-Gaussian time series [20]. In turn, we focus on a subset of possible approaches that will be further utilized in the course of this work in an application to some exemplary radon time series.

\subsection{ARFIMA modelling}

One way of characterizing possible long-range dependence in observational time series is fitting some appropriate stochastic process model to the data which may or may not exhibit this kind of variability. A natural choice for a class of such models are autoregressive fractionally integrated moving average (ARFIMA) models, which have the form [19]

$$
\alpha(B)(1-B)^{d} X_{t}=\beta(B) \varepsilon_{t}, \quad 0<d<\frac{1}{2},
$$

where $d$ is the order of fractional differentation characterizing the memory of the process, $B$ is the backshift operator $\left(B X_{t}=X_{t-1}\right), \alpha(B)$ and $\beta(B)$ are two polynomials of degrees $p$ and $q$, respectively, represented in terms of powers of the backshift operator applied to the process $\left\{X_{t}\right\}$, and $\left\{\varepsilon_{t}\right\} \sim \mathcal{N}\left(0, \sigma_{\varepsilon}^{2}\right)$ represents a standard Gaussian 


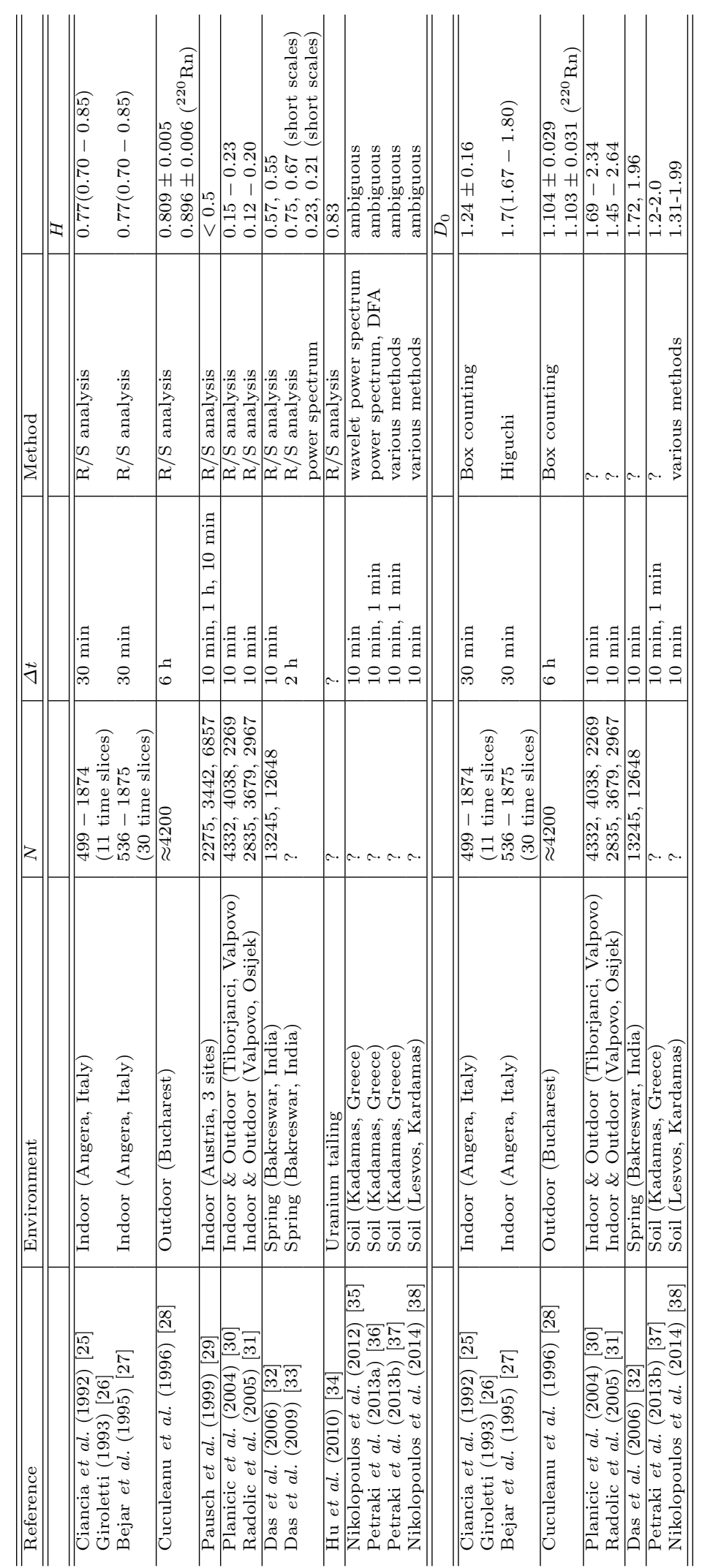

Table 1. Selection of recent results on possible long-range dependence and fractal scaling in ${ }^{222} \mathrm{Rn}$ time series from different environments. 
white noise process with zero mean and variance $\sigma_{\varepsilon}^{2}$. The fractional differentiation can be expressed in terms of a series expansion

$$
(1-B)^{d}=\sum_{k=0}^{\infty} \frac{\Gamma(d+1)}{\Gamma(k+1) \Gamma(d-k+1)}(-1)^{k} B^{k} .
$$

Specifically, if $d$ is non-integer the underlying process exhibits long-term memory. For stationary series, $d \in(-0.5,0.5)$, and the Hurst exponent associated with the process is given by $H=(2 d+1) / 2$. Consequently, long-range memory is present for $d \in(0,0.5)$, while $d \in(-0.5,0)$ indicates anti-persistent fluctuations.

In this work, parameter estimation of ARFIMA models for the observed radon time series is based on the classical maximum-likelihood principle approximated by the Haslett-Raftery method [44] using the implementation in the R package fracdiff. The validity of the model can be tested using classical model selection approaches like likelihood ratio tests or penalized-likelikood criteria (e.g., the Akaike or Bayesian information criteria) by comparing the goodness-of-fit of a model with free parameter $d$ with that of an alternative model where $d$ is kept fixed at an appropriate integer value (ARIMA model) or $d=0$ (ARMA model).

\subsection{Spectral analysis}

While the ARFIMA model parameter $d$ provides a quantitative characterization of long-range memory, the underlying assumption of a specific parametric model can be quite restrictive. Therefore, the most common approach in environmental sciences is directly estimating the Hurst exponent $H$, which can be obtained without making explicit assumptions on the underlying model structure (however, most available estimators typically impose certain assumptions implicitly). Among others, a widely applicable strategy (which is superior to many other approaches in rather general cases [20]) is based on the observation that short- and long-term correlations lead to distinctively different scaling properties in Fourier space due to the link between power spectral density (PSD) and auto-correlation function via the Wiener-Khinchine theorem. Specifically, in case of long-range dependent time series, the PSD exhibits a power-law behavior with a characteristic exponent $\beta$ related to the Hurst exponent of the process via $H=(\beta-1) / 2$. Stationarity requires $1<\beta<3$ corresponding to $H \in(0,1)$.

For the purpose of estimating the PSD, there is a variety of methods available. In this work, we will restrict ourselves to two approaches: the time-averaged wavelet spectrogram (i.e., the mean of the time-frequency unfolding of the signal by means of continuous wavelet transform, CWT, evaluated individually at all time-scales) [4547] and the maximum taper method (MTM) [48]. We refer to the corresponding references for detailed discussions of both methods. To this end, we only emphasize that the MTM is well suited for identifying individual periodic components contained in a time series with high precision and confidence, whereas the CWT-based methods shares the property of an intrinsic (time-)frequency uncertainty generically shown by all wavelet-based methods and therefore provides a smooth spectral estimate rather than great detail in the frequency domain.

As an important note, we emphasize that estimating $H$ from the PSD requires assessing the low-frequency behavior. However, the latter is usually affected by the finite time series length and therefore provides insufficient information for obtaining a proper estimate. In turn, assuming that the studied process obeys a consistent scaling over the whole spectrum, $\beta$ is commonly obtained via regression of a power-law model fitted to the high-frequency tail of the spectrum. 


\subsection{Fractal dimensions}

Long-range dependence is typically accompanied by fractal scaling properties of the time series. Specifically, for certain types of stochastic processes the fractal dimension $D_{0}$ of the geometric object formed by the graph of a time series (which is to be clearly distinguished from the fractal dimension of a dynamical system generating this series $[49,50])$ is known to exhibit a unique relationship with the Hurst exponent. Specifically, for a univariate time series, $D_{0}=2-H$. However, this relationship is not unique and typically assumes the presence of a fractional Brownian motion (fBm) or related process, whereas it breaks down for other processes [51]. Nevertheless, independent of a specific process model, calculating the fractal dimension from time series can give complementary information about the scaling properties of the process, which are commonly not independent from its dynamical persistence.

In this work, we use two different estimators of the fractal dimension $D_{0}$ : a boxcounting algorithm designed for application to one-dimensional time series data [52] and the celebrated Higuchi method $[53,54]$. Notably, we do not discuss other types of fractal dimensions like information $\left(D_{1}\right)$ or correlation dimension $\left(D_{2}\right)$, which could further reveal possible multifractality. However, the latter aspect will not be further discussed in this paper.

\subsection{Detrended fluctuation analysis}

Many classical methods for estimating the Hurst exponent from time series data are based on the evaluation of certain scaling characteristics arising from long-term persistence. Besides the classical R/S analysis and the estimation of the scaling exponent of the PSD, one of the most widely used methods is detrended fluctuation analysis (DFA) [55]. Given its simple implementation and wide-spread use in various fields of science, we will utilize this method in the present work as well, being aware that systematic studies have revealed that methods based on the PSD are commonly superior to DFA in terms of bias and variance of the estimated Hurst exponents [20].

Unlike R/S analysis, DFA is based on evaluating the scaling characteristics of the time series profile $Y_{t}=\sum_{\tau=1}^{t} X_{\tau}$ (here, the process $\left\{X_{t}\right\}$ is commonly transformed to zero mean prior to computing the profile), implicitly assuming that $\left\{Y_{t}\right\}$ is described by an (intrinsically non-stationary) fBm model. Specifically, $\left\{Y_{t}\right\}$ is divided into $\lfloor T / w\rfloor$ non-overlapping boxes of $w$ points each. Next, the local trend $z_{t}^{(k)}$ (where $k$ indicates the box number in the sequence) within each box is removed by fitting a polynomial of order $n, z_{t}^{(k)}=\sum_{p=0}^{n} a_{p}^{(k)} t^{p}$, to the data within the $k$-th box. The order $n$ of $z^{(k)}$ is referred to as the order of DFA. $n=0$ corresponds to classical fluctuation analysis suitable for stationary data, whereas $n>0$ is recommended for non-stationary series [56].

The characteristic scaling property utilized by DFA is based on the detrended fluctuation function

$$
F_{k}^{2}(w)=\frac{1}{w} \sum_{t=k w+1}^{(k+1) w}\left|Y_{t}-z_{t}^{(k)}\right|^{2}, \quad k=0, \cdots,\lfloor T / w\rfloor-1
$$

Averaging $F_{k}^{2}(w)$ over the $\lfloor T / w\rfloor$ intervals gives the fluctuation $F(w)$ as a function of the window size $w$ :

$$
F(w)=\left\{\frac{1}{\lfloor T / w\rfloor} \sum_{k=0}^{\lfloor T / w\rfloor-1} F_{k}^{2}(w)\right\}^{1 / 2}
$$


If the original variable $X_{t}$ exhibits long-range correlations, the detrended fluctuation function follows a power-law

$$
F(w) \sim w^{H} .
$$

By definition, DFA avoids possible artifacts due to inherent trends of arbitrary order (and, due to the discrete sampling of the data, present at different time scales) in the data $[57,56]$. Specifically, by increasing the DFA order $n$, trends at different scales are effectively removed [56], so that the effect of local correlations can be easily probed [58].

\section{Description of the data}

The concentration of the noble gas radon tends to build-up in indoor environments, particularly in basements and poorly ventilated places. In order to assess the indoor radon concentration in an unventilated archive room at the Faculty of Sciences and Technology of the University of Coimbra, the radon concentration was continuously monitored in a closed room located at the floor level and in direct contact with sandstones of Triassic age.

The monitoring set-up has already been described in detail by Neves et al. [8]. The radon concentration was measured in terms of alpha particles counts during 5minutes intervals within a period of about 6 months, from 1 March to 18 September 2007. For these measurements, a RM-80 detector (Aware, USA) sensitive to gamma radiation and $\mathrm{X}$-rays above $10 \mathrm{keV}$ was used. The system was calibrated in a calibration chamber (Genitron, Germany) with a certified radon source NIST SRM-4973 (National Institute of Standards \& Technology, USA). Furthermore during one week, an AlphaGUARD monitor was operated simultaneously with the RM-80 detector in the room selected for the study in order to establish a relation between the average radon concentration and average counts per minute registered by the two instruments. Based on the obtained detector characteristic of $0.516 \mathrm{~Bq} \mathrm{~m}^{-3}$ per cpm (counts per minute), the measured particle counts were transformed to $\mathrm{Bq}^{-3}$ [8].
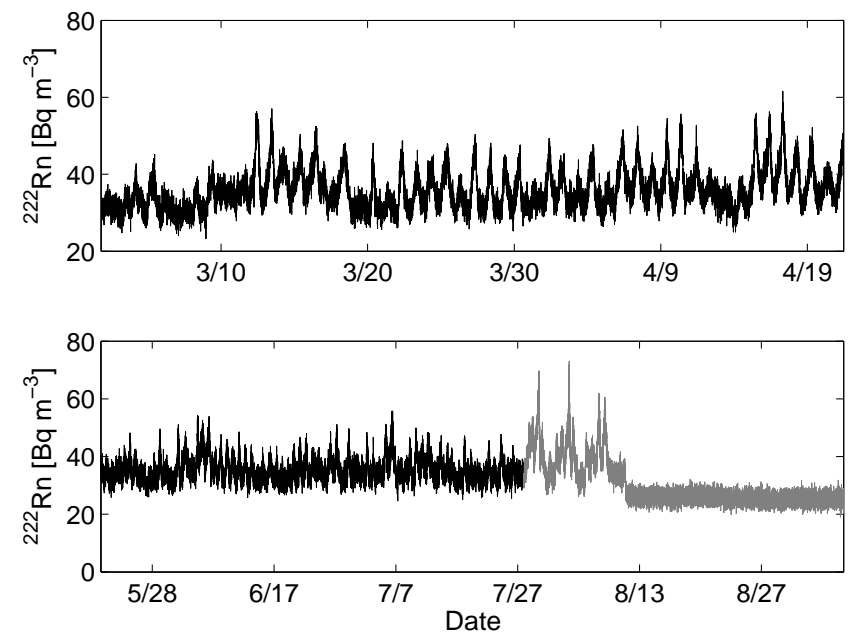

Fig. 1. Time series of indoor radon concentrations used in this study: COI1 (top), COI2 (bottom). The gray part of COI2 is not utilized. 


\begin{tabular}{l|c|c}
\hline \hline & COI1 & COI2 \\
\hline \hline Mean & 35.8541 & 35.1861 \\
Standard deviation & 5.1620 & 4.0975 \\
Median & 34.8816 & 34.4688 \\
Mean absolute deviation & 4.0328 & 3.1509 \\
Skewness & 0.9238 & 1.0116 \\
Kurtosis & 3.9583 & 4.3441 \\
\hline \hline
\end{tabular}

Table 2. Basic statistical properties of the two radon time series used in this study.

Continuous measurements were interrupted for about four weeks (from 21 April to 19 May) due to an instrumental failure (Fig. 1). Moreover, data recorded after 28 July 2007 have been discarded because of a marked change in the behavior of the radon variations following a change in the measurement setup (gray part in Fig. 1). Accordingly, we split the available data into two time series with a temporal resolution of 5 minutes, corresponding to the periods before 21 April 2007 and after 19 May 2007 $(N=14,591$ and 19, 999 data points, respectively). In what follows, these subsets will be referred to as COI1 and COI2. The very detailed temporal resolution and the resulting lengths of the available time series permits the utilization of modern nonlinear techniques of time series analysis [59]. Some basic statistical properties of the two data sets are summarized in Tab. 2.

The data were previously examined by Neves et al. [8], focusing on the periodicities embedded in the records (particularly the diurnal cycle) and on the investigation of their possible drivers. The study concluded that the daily patterns were mainly influenced by the outdoor temperature and by rainfall.

It is important to note that the two subseries COI1 and COI2 are continuous and complete, i.e., both are not affected by missing values due to measurement interruptions, instrumental malfunctions, datalogger failures or similar problems commonly appearing in environmental recordings. Otherwise, there would be two possible strategies to cope with such problems in the context of the present work unless the corresponding gaps become too large. On the one hand, there are sophisticated gap-filling approaches that allow imputing missing parts of the time series while maintaining the underlying multi-scale correlation structure [60,61]. On the other hand, missing data can be either ignored in the evaluation of sums (as in the case of DFA) or explicitly accounted for by specifically tailored estimators (such as the Lomb-Scargle periodogram for the PSD).

\section{Long-range dependence and fractal scaling}

In this section, we report the results of the different analysis techniques detailed in Section 2 when naïvely applied to the high-frequency radon time series described in Section 3. As we will see, this "black-box" strategy leads to ambiguous and potentially hard-to-interpret results. The underlying conceptual problems, that likely apply to some previous studies on long-term persistence in radon time series, are further detailed.

\subsection{Basic signal properties}

As briefly mentioned in Section 2, some estimators of the Hurst exponent can show a systematic bias and increased variance when applied to data with non-Gaussian 
shape. Specifically, the existence of strong deviations from a Gaussian indicates that a $\mathrm{fBm}$ cannot be an appropriate model for explaining a possible long-range memory in the data. In fact, this is the case for both COI1 and COI2 (Fig. 2), which exhibit a strongly asymmetric and positively skewed PDF. Still, a fractional random walk-like process with a non-Gaussian distribution could be compatible with the observed data structure.

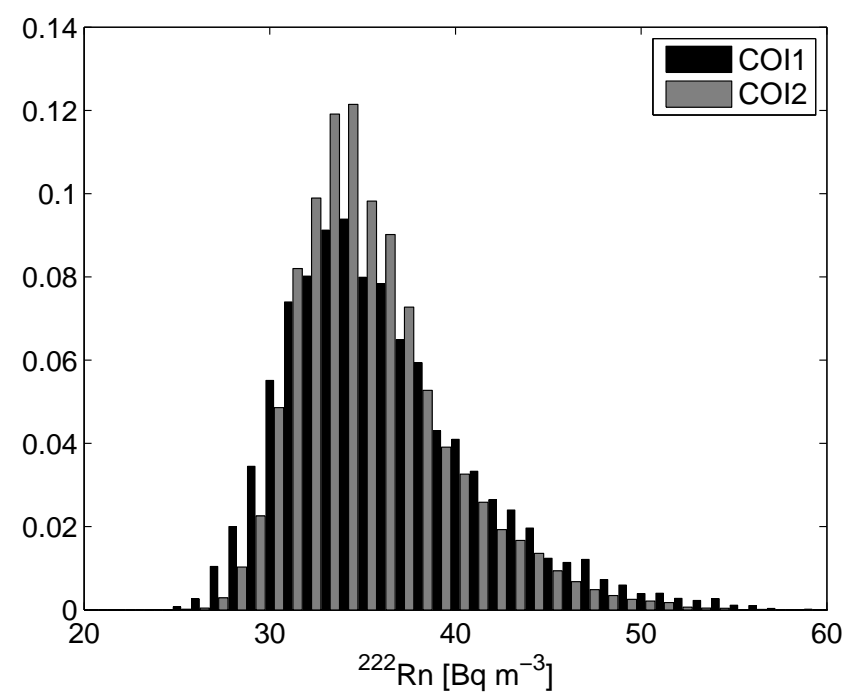

Fig. 2. Histogram of the indoor radon concentrations for the COI1 (black) and COI2 (red) data sets.

In order to get a first impression on whether or not a possible extension to a multifractal process might be necessary, we additionally consider the increments of radon fluctuations with different time lags (Fig. 3). Unlike the radon concentrations themselves, the increments exhibit a clearly symmetric distribution without indications of heavy (power-law shaped) tails, suggesting that the intermittency often observed in multifractal processes $[62,63]$ is missing in these data. We emphasize that this is no proof of the absence of multifractality, but provides some first hints that a description by a classical mono-fractal process might be sufficient for the considered radon time series.

\subsection{ARFIMA models}

Fitting parametric models to the two time series segments clearly suggests the presence of long-range memory in the time series. Specifically, for a simple fractional differences (FD) model (an ARFIMA model with the auto-regressive and moving average orders $p=q=0$ ) fitted to the raw data, we find fractional orders of $d=0.468 \pm 0.001$ and $d=0.423 \pm 0.001$ for COI1 and COI2, respectively, pointing to long-range correlated behavior. However, we also observe that the fractional orders found for both subsets do not match each other, which could be related to non-stationarity in the correlation structure of the considered radon variations.

The results become less obvious when allowing for nontrivial coefficient polynomials $\alpha(B)$ and $\beta(B)$, i.e., considering the full class of $\operatorname{ARFIMA}(p, d, q)$ models. For 


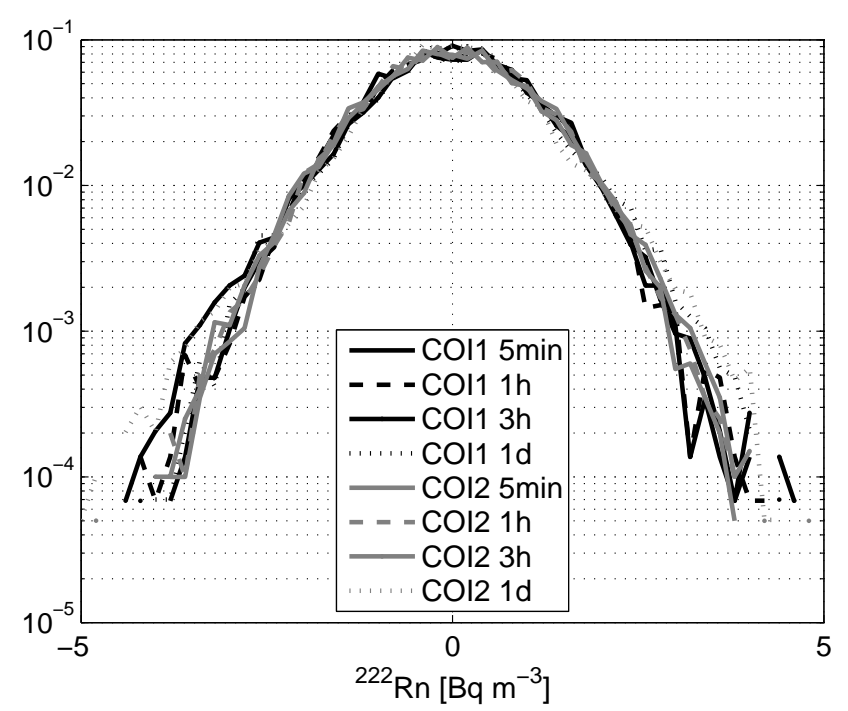

Fig. 3. Histogram of the increments the indoor radon concentrations for the COI1 (black) and COI2 (grey) data sets for different time lags between 5 minutes and 1 day.

convenience, we apply linear detrending to the radon time series prior to model estimation, and select the best models according to the Akaike Information Criterion. In this case, model selection recommends an $\operatorname{ARFIMA}(1, d, 2)$ model with $d=0.20$ for COI1, and an ARFIMA $(2, d, 1)$ model with $d=0.061$ for COI2, suggesting that the possible long memory is more strongly expressed in COI1 than COI2. Comparing the power spectral densities of the observed radon time series with those of the FD and full ARFIMA models (Fig. 4), we conclude that the more complex models with less expressed long-range dependence fit the distribution of spectral power better than simple long-range dependent FD models.

\subsection{Power spectral density}

For studying the dynamical characteristics of the observed radon concentrations in some more detail, we next turn to the corresponding PSD. Figure 5 shows the estimate based on the time-averaged wavelet spectrogram. It is clearly seen that the spectrum does not exhibit a clear power-law shape in the high-frequency part, but reveals rather distinct behaviors within different frequency ranges.

At high frequencies corresponding to periodicities of up to about one hour, the spectrum is essentially flat (note that the decay shown by the wavelet-based estimate at the highest frequencies is mainly an artifact of the method and is not revealed when considering, e.g., the MTM estimate). This implies that the high-frequency variability in the radon time series essentially corresponds to a stochastic process without memory (i.e., white noise). The most likely interpretation of this finding is that the overall level of environmental radioactivity at the study site is comparably low, so that there are too few alpha counts originating from radon exhalation within each five-minutes measurement interval to actually represent a meaningful signal. This finding also supports the setting previously used in [8] where the signals were integrated over one hour periods prior to further analysis.

For time scales between about 3 hours and 1 day (the peak associated with the diurnal cycle of radon variations), the spectrum displays a marked decay. Naïve es- 

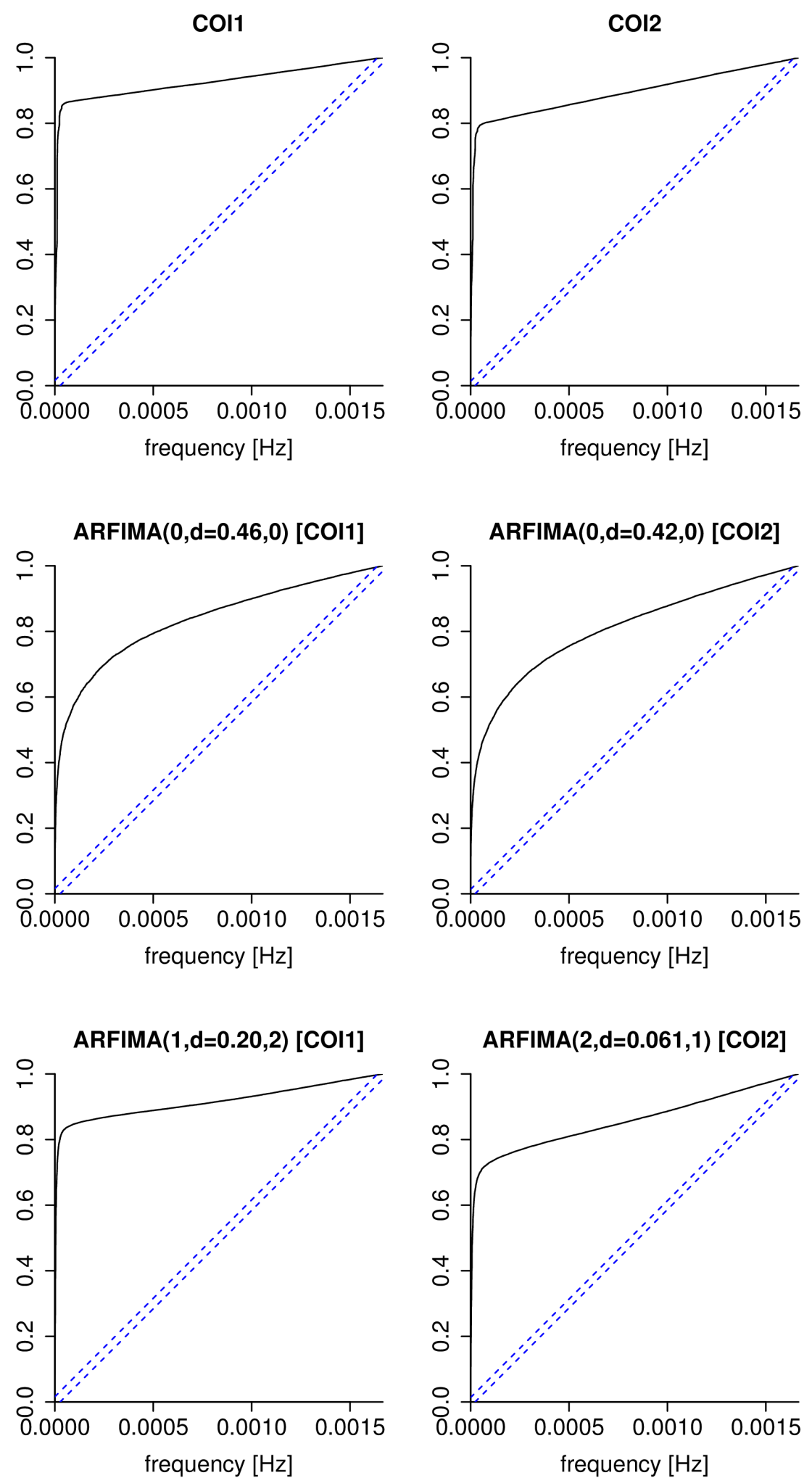

Fig. 4. Cumulative periodograms of the original data, FD and best ARFIMA models (from top to bottom) for COI1 (left) and COI2 (right), indicating that an appropriate representation of the distribution of spectral power among different frequencies requires the more complex models with less indication of long-range memory. 


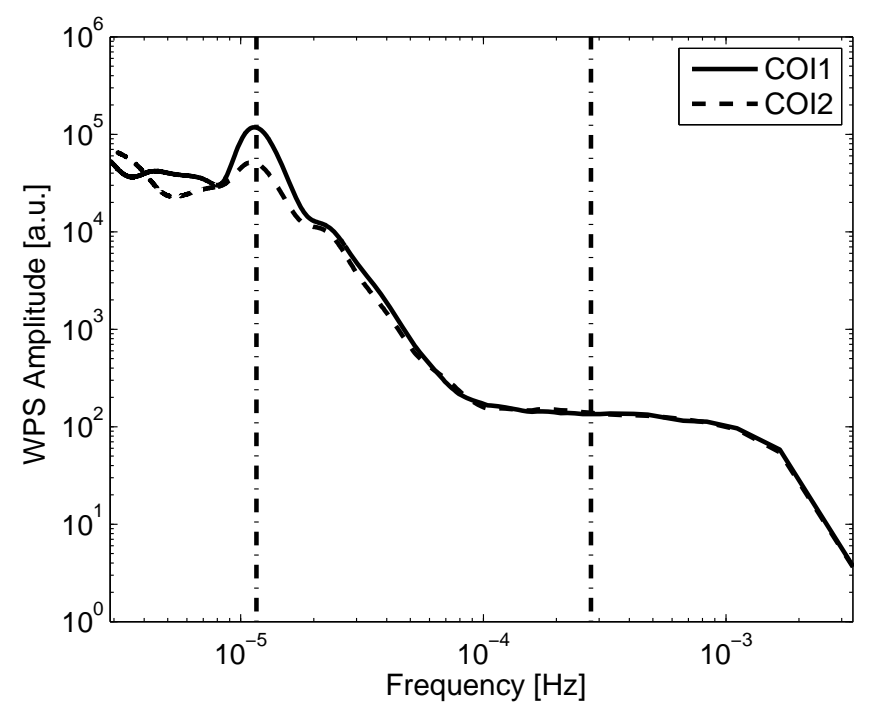

Fig. 5. Wavelet power spectrum (WPS) amplitudes (defined as time-averages of the associated wavelet spectrograms inside the respective cones-of-influence obtained with a complex Morlet mother wavelet) as smooth estimates of the power spectral density of the COI1 (solid, black) and COI2 (dashed, gray) time series. Vertical (dash-dotted) lines indicate 1-day (left) and 1-hour (right) periods, respectively.
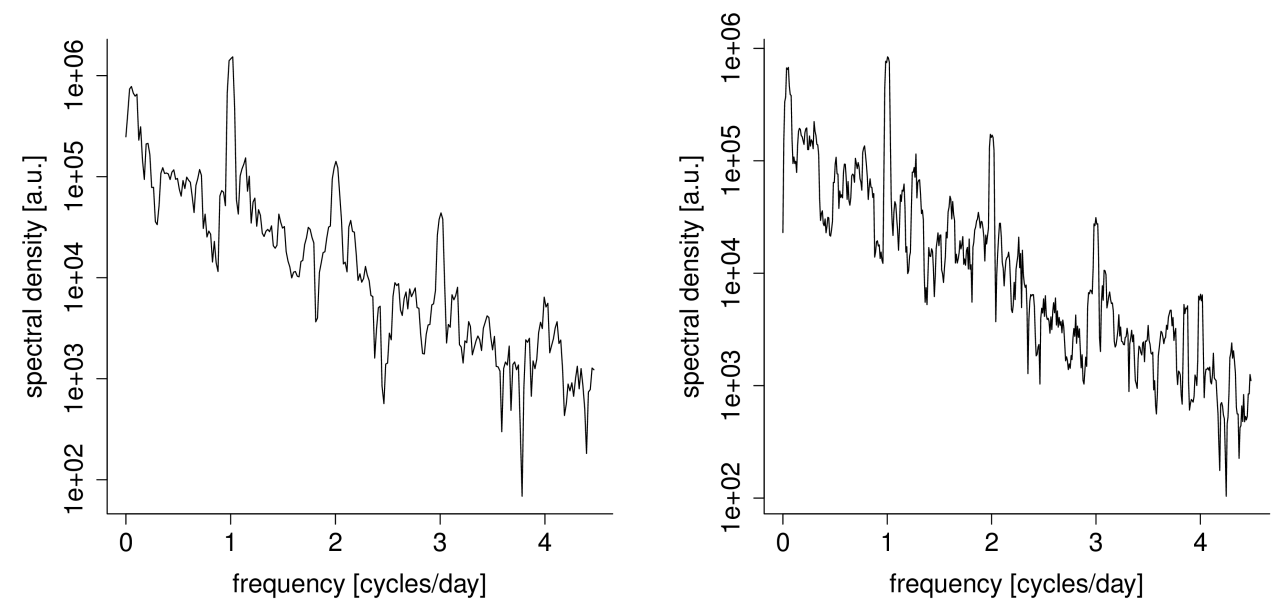

Fig. 6. MTM estimates of the power spectral density for the COI1 (left) and COI2 (right) time series using three eigentapers to reduce the variance of the spectral estimates [48].

timation of the associated slope for periods clearly below 1 day gives values $\beta>3$ exceeding the limit for stationary processes. However, this result is not to be overemphasized, since utilizing the alternative MTM estimator (providing a higher frequency resolution) reveals a sequence of smaller peaks in the spectrum (Fig. 6). The latter finding is consistent with previous results demonstrating the presence of additional periodicities of about 12, 8, 6 hours, etc., in the Coimbra data [8] as well as other environmental radon records [64-67]. Taken together, the part of the spectrum between about 3 hours and 1 day should not be used for estimating a spectral slope 
associated with possible long-range memory, since it mainly reflects the presence of periodic components in the signal.

Finally, the low-frequency range comprising periodicities of more than one day is the only part of the spectrum that might be used for assessing the possible presence of long-range correlations in Radon. However, there are two problems associated with this strategy. On the one hand, regarding the longer time-scales the available data are comparably short, which implies that we may expect a large variance of spectral estimates of the Hurst exponent. On the other hand, the dominant presence of the diurnal peak in the spectrum can lead to a systematic bias of the spectral slope compared with what a similar signal without diurnal cycle would exhibit. We will revisit this aspect below.

\subsection{Detrended fluctuation analysis}

The analysis of the PSD of the considered radon time series has revealed a distinctively different behavior at different time scales. In the following, we demonstrate that the same information is also manifested in the detrended fluctuation functions. Figure 7 displays the corresponding results for both time series. As can be clearly seen, the observed behavior of $F(w)$ is - in doubly-logarithmic scale - far away from being linear as one would expect for a "normal" long-range dependent process. Instead, we can again distinguish three different ranges of scales:
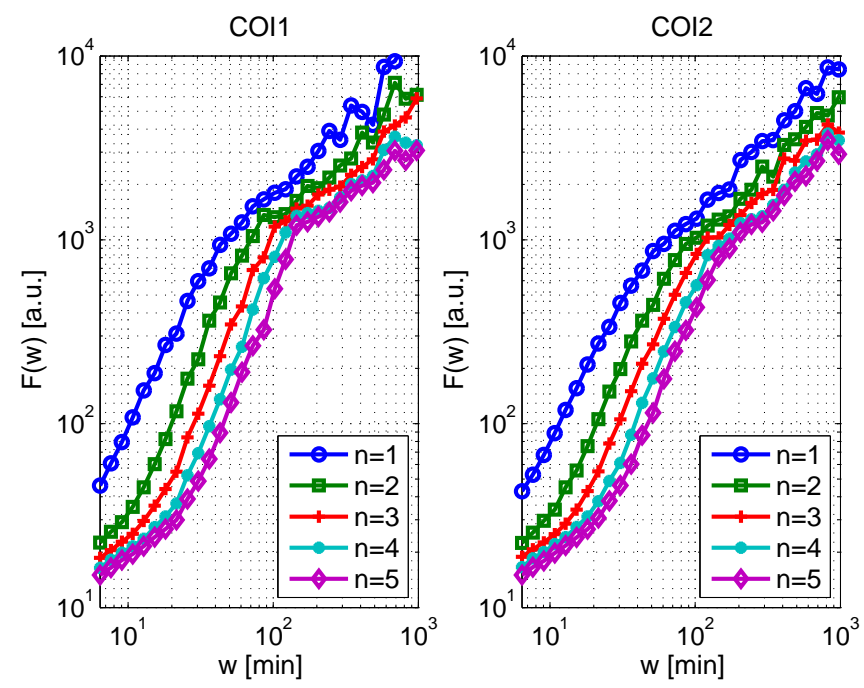

Fig. 7. (Color online) Detrended fluctuation functions $F(w)$ in dependence on the scale $w$ for different orders $n$ of detrending for the COI1 (left) and COI2 (right) time series.

First, time-scales corresponding to high-frequency variations show a very shallow increase of the fluctuation function with a slope of the order of 0.5 , which is compatible with classical Brownian motion (resulting from aggregating Gaussian white noise). The upper cutoff of this range increases with increasing order of detrending, indicating that the detrending by higher-order polynomials effectively removes the impacts of the oscillatory components down to time-scales of several hours. Conversely, we emphasize that when filtering the observed cycles as well as all low-frequency variability beyond 
time-scales of one day, the resulting time series might essentially show the features of short-term correlated records.

Second, intermediate scales (with the exact range depending on the DFA order) are characterized by a steep increase of the fluctuation function with a slope of about 1.5 (depending on the exact range of time-scales for which the slope is estimated). Taking this regime for obtaining a scaling exponent, the conclusion would be that the underlying process is non-stationary. In turn, the correct interpretation of this finding is that the dominance of the diurnal cycle - together with semi-diurnal cycle and other sub-diurnal oscillations - dominates this frequency range [8], and what is seen by the fluctuation function (similar as for the PSD) is essentially the superposition of broad spectral peaks corresponding to these oscillatory modes plus possible sidebands [66].

Finally, at time-scales beyond 1 day, the fluctuation function shows again a shallower slope, which would correspond to the scales at which we might expect long-range memory to be detectable. Taking this slope for estimating the Hurst exponent yields values of the order of $H \sim 0.8$ compatible with this expectation, i.e., providing an indication for long-range correlations being actually present in the signals. Unfortunately, with the available data, this scaling can only be traced up to about 20 days, since for longer scales that amount of data is far too small, leading to a saturation of the fluctuation function. Notably, the problem of properly addressing any kind of scaling over such a small range of time-scales is not intrinsic to DFA, but also shared by all other time series analysis techniques utilized in this study and beyond. Therefore, longer time series appear necessary to unambiguously detect the possible presence of long-range memory. However, in many real-world situations, such longer records are not available (e.g., due to the general costs of extended measurement campaigns or instrumentation problems), so that we consider the conditions met in the present example a rather typical case in environmental radioactivity studies.

We emphasize that the emergence of different scaling regimes in the fluctuation function is a common effect when studying time series with monotonous or even low-frequency oscillatory trends. Specifically, the presence of periodic components necessarily results in artificial slopes of the fluctuation function. A detailed discussion of the corresponding effect is provided in $[56,57]$.

\subsection{Fractal dimensions}

For the sake of completeness, we report the results for the two different estimates of the fractal dimensions discussed in Section 2. Notably, both box-counting and Higuchi method again make use of scaling characteristics, i.e., they evaluate some statistical property in dependence on the level of coarse-graining applied to the time series under study. As for PSD and DFA, we find that the resulting functional dependences on the scale are not perfectly linear in doubly-logarithmic representation (not shown), but exhibit less dependence on the specific scale so that an overall linear fit appears justified. As a result, we obtain estimates of the fractal dimension of 1.78 (1.8) for COI1 (COI2) with the box-counting method, and 1.93 (1.95) with the Higuchi method. Note that under the classical assumption of a fBm process, these values would imply an anti-persistent process $(0<H<0.5)$, which is in sharp contrast with the findings based on PSD and DFA. We therefore conclude that (unlike frequently assumed in the literature) the observed variability is of a type that is not appropriate for studying long-range dependence based on fractal dimension estimates. We emphasize that similar apparently (i.e., when sticking to the fBm hypothesis) inconsistent results have been found in at least one other previous studies [27], while others reported values matching $H=2-D[25,26,28]$. 


\section{Separation of essential and background variability}

The results of spectral analysis and DFA presented in Section 4 (but also those of other complementary methods making use of scaling properties of the time series, e.g., R/S analysis - not shown here) suggest that the inherent scaling properties of the radon time series depend crucially on the considered range of time-scales, which is predominantly due to the strong oscillatory modes in combination with the relatively low average level of environmental radioactivity dominating the behavior in the highfrequency part of the signal. There appear to be two potential strategies for coping with this situation.

On the one hand, one could accept the Hurst exponent as being a scale-specific characteristic and use the PSD or DFA results for estimating scale-based Hurst exponents [68] from the local slope of the PSD or fluctuation function, respectively. However, due to the limited amount of data and the generic variance of scale-local estimates, we expect this procedure to result in numerically rather unstable values. Therefore, this idea will not be further considered here, but shall be addressed in more detail in future work.

On the other hand, we emphasize that the problems with identifying and quantifying possible long-range persistence in radon time series are mainly due to the multi-scale dynamics characterized by various oscillatory components at different time-scales (diurnal, semi-diurnal, etc.) as well as the complex multi-day variability. Therefore, time-scale decomposition for (i) removing the oscillatory components and (ii) filtering the noisy high-frequency contributions prior to further analysis appears an intuitive strategy. In this section, we will further explore this potential approach.

\subsection{Time series decomposition by singular spectrum analysis}

There exist a multiplicity of techniques for decomposing time series into components corresponding to oscillatory variability patterns with more or less distinct natural frequencies. In the context of radon time series, wavelet methods $[9,69,70]$, singular spectrum analysis (SSA) $[42,43]$ as well as empirical mode decomposition (EMD) [41] have been applied in recent studies. Among these techniques, discrete wavelet analysis [9] has the potential disadvantage of providing a decomposition into frequency bands rather than signals with specific periodicities. In turn, continuous wavelet analysis $[69,70]$ provides such specific components, but on the cost of the reconstruction of the signal after removing selected components being a challenging task. Finally, EMD is known to be powerful when applied to signals exhibiting oscillations with time-varying frequencies, but can display mixed modes if distinct but close frequencies are contained in the signal under study. As a consequence, in what follows we will restrict our attention to SSA for decomposing our radon time series. Since both series of indoor radon concentrations from Coimbra are apparently very similar (see Section 4), we will only show the results for the COI1 time series.

Figure 8 shows the variances of the reconstructed SSA components (corresponding to the eigenvalues of the Toeplitz matrix containing the lagged auto-correlation coefficients of the underlying time series). It is evident that three components clearly stand out of the continuum of eigenvalues, implying that three SSA modes appear to provide a reasonable approximation of the original time series. This finding is supported by Fig. 9 which shows the original time series together with the reconstruction obtained by a superposition of the first three SSA modes weighted by the associated eigenvalues. As can be seen, the reconstruction nicely traces the diurnal and multi-day variability, while the high-frequency (supposedly noisy) variations are effectively removed from the signal. Our corresponding results are consistent with the 


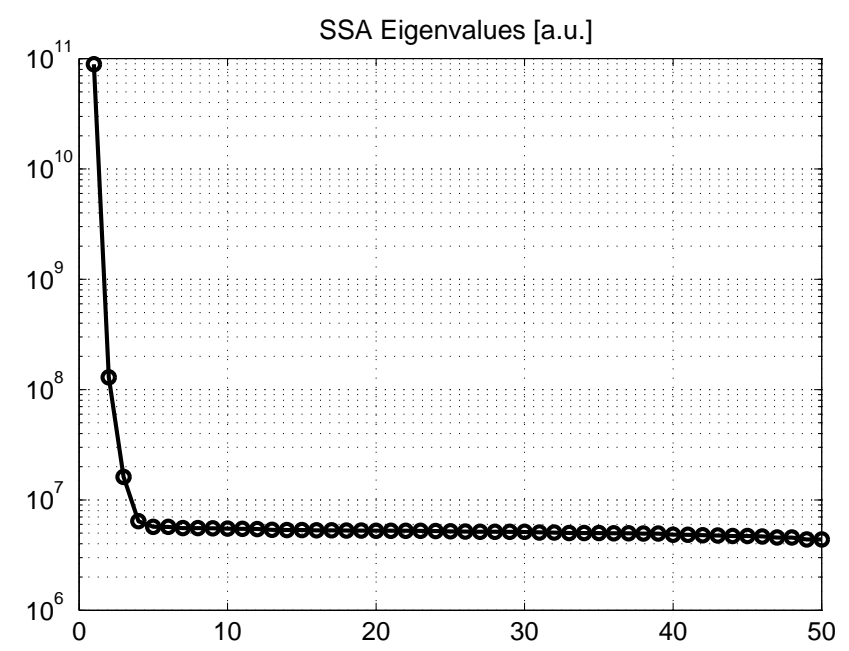

Fig. 8. SSA eigenvalues (variances of the reconstructed SSA components) for the COI1 data set.

fact that the reconstruction of strong periodic components (in our case, the diurnal cycle) requires a pair of SSA eigenmodes, whereas the third SSA component appears to mainly reflect the multi-day variations (i.e., the supposed carrier of possible longrange dependence).
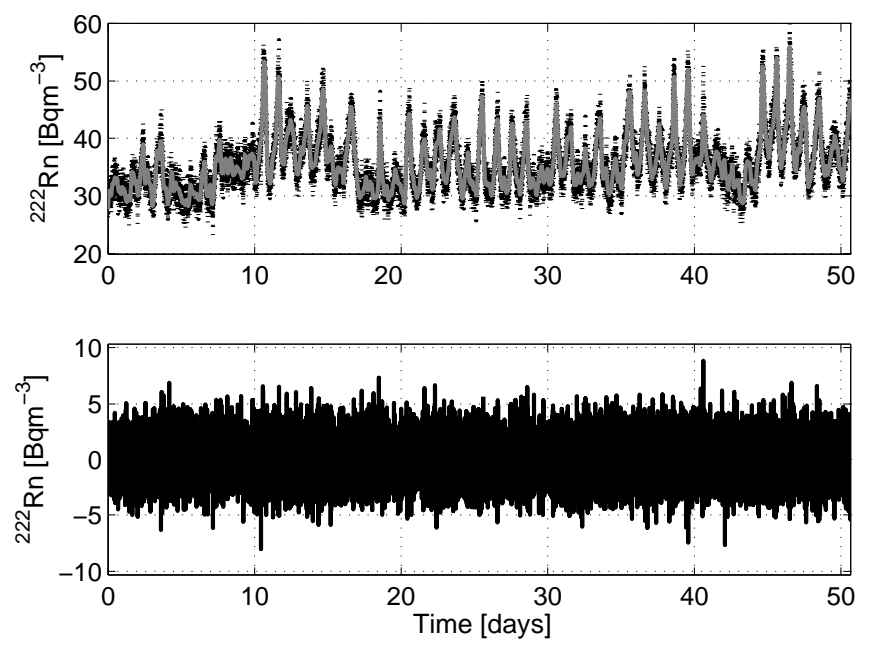

Fig. 9. Top: Original time series (black) and reconstruction based on the first three SSA components (grey) for the COI1 data set. Bottom: Residual variability after subtraction of the first three SSA components from the original signal. 


\subsection{Filtered time series: Power spectral density}

In order to further demonstrate that the proposed decomposition provides a reasonable approximation to the original data, we consider the PSD of the thus reconstructed (aka filtered) signal and the "background variability" (i.e., the superposition of all remaining SSA components). Note that we have used a finite number of $m=50$ SSA components in this example. Consequently, there is still some residual variability not explained by the 50 considered components; however, the variance of this residual is of the order of the numerical accuracy of our computations and does not contain any systematic temporal structure.

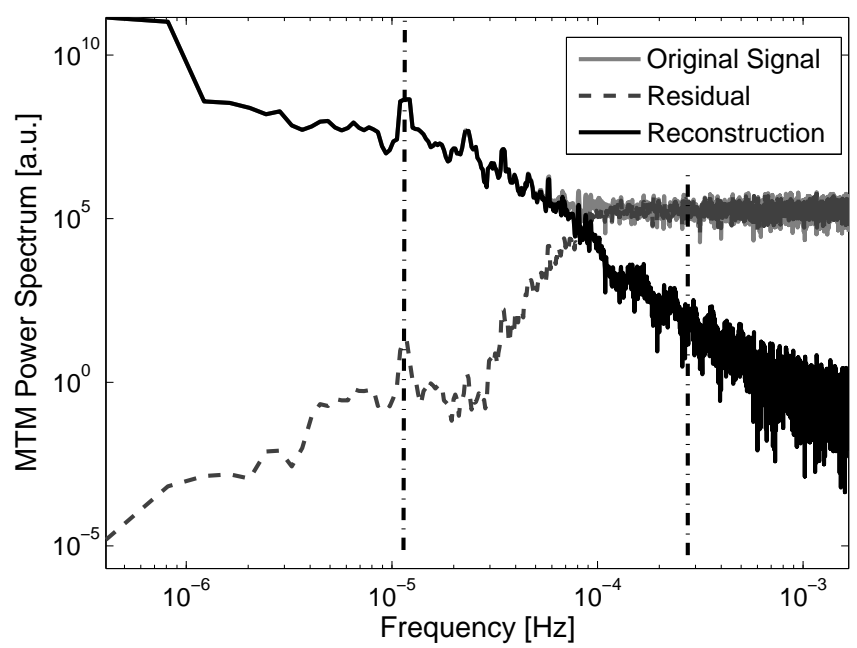

Fig. 10. MTM estimates of the PSD of the COI1 time series: original data (grey), reconstructed signal consisting of the first three SSA components (solid, black), and residual (dashed, black). In all cases, six discrete prolate spheroidal sequences have been employed as data tapers together with a fast Fourier transform of length $N=512$. Vertical (dash-dotted) lines indicate 1-day (left) and 1-hour (right) periods, respectively.

Looking at the resulting PSD of SSA reconstruction and residual, a couple of observations are made:

On the one hand, the reconstruction displays the same sub-diurnal fluctuations (e.g., the semi-diurnal cycle) as the original data, suggesting that the presence of diurnal and sub-diurnal periodicities with integer frequency ratios might be coupled to each other due to the nonlinearity and heteroskedasticity of the data. A more detailed study of this aspect is beyond the scope of this work. In addition, we also observe that the residual still exhibits a clear diurnal cycle as well as sub-diurnal periods (although being orders of magnitude weaker than in the original signal), implying that the SSA decomposition (based on linear transformations of the data) is not capable of fully removing these oscillations from the (nonlinear) signal.

On the other hand, the residual essentially contains the high-frequency variability of the original radon time series, with time-scales below one hour essentially showing a flat spectrum corresponding to a white noise process. In turn, for the SSA reconstruction this high-frequency part exhibits a steep power-law slope. 


\subsection{Implications for inferring long-term memory}

From the previous results, we conclude that classical time-scale decomposition techniques may not be capable of properly removing undesired components hampering the study of long-range persistence in the data. In the present example, it is possible to eliminate the high-frequency noise from the data, however, the same could be achieved by just aggregating the data to hourly values as done in the previous work by some of the authors [8]. Hence, SSA leaves the choice of continuing with a signal that still exhibits diurnal as well as sub-diurnal cycles or the white noise background, both of which are not helpful in our problem of inferring the presence of long-term correlations from the data.
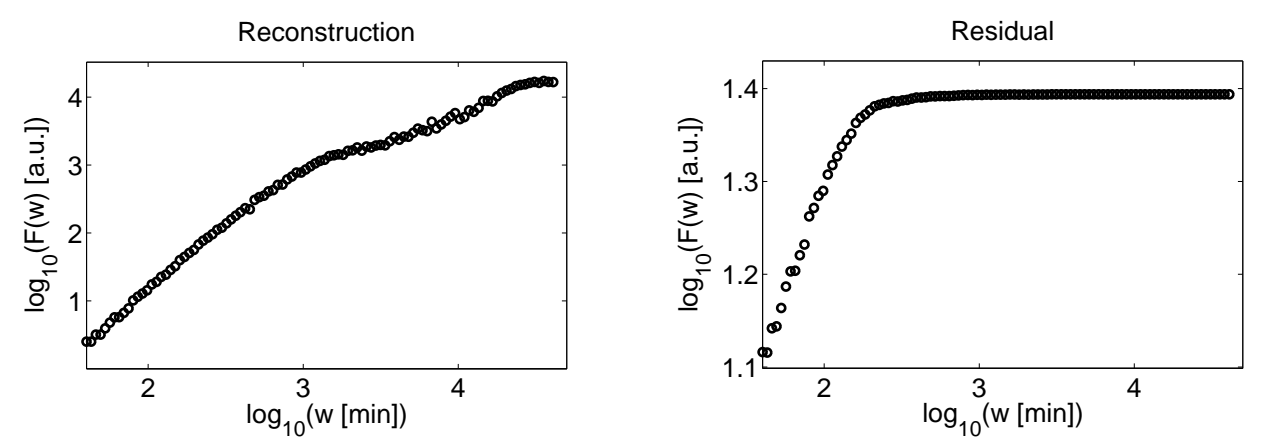

Fig. 11. Detrended fluctuation function of the reconstructed signal based on the first three SSA modes (left) and the corresponding residual (right).

In Fig. 11 we exemplify this situation for the case of DFA. Specifically, considering the behavior of the detrended fluctuation function for SSA reconstruction and residuum, we find that in both cases two characteristic scalings remain. For the residuum, we have find a slope (in doubly-logarithmic representation) of 0.5 at short scales (corresponding to the high-frequency continuum characterized by an absence of correlations), while the rest of the fluctuation function essentially remains constant for larger scales above about 5-6 hours. In turn, for the reconstruction there is a steep slope of about 1.9 for time-scales below about 1 day, indicating again the apparent non-stationarity of the signal induced by the diurnal and sub-diurnal variability components, whereas we observe a slope of $H \approx 0.84$ for longer time-scales. We expect this slope to represent the "true" long-range dependence of the observed radon time series.

Similar findings are made for the fractal dimension estimates. For example, when applying the box-counting method to the SSA reconstruction of the COI1 data set, we find two slopes with exponents of 1.88 and 1.19, respectively, the latter approximately matching the estimate of the Hurst exponent from DFA under the fBm assumption.

\section{Conclusions}

We have demonstrated that typical features of radon time series, predominantly their multi-scale dynamics in combination with heteroskedasticity and, in general, nonlinearity systematically hampers the investigation of long-range memory in environmental radioactivity fluctuations. Our results for two time series of indoor radon concentrations from Coimbra (Portugal) have illustrated that a naïve application of 
time series analysis methods for studying long-range dependence and fractal scaling is prone to provide misleading results if the multi-scale nature is not appropriately taken into account. Specifically, different time-scales need to be carefully distinguished: (i) high-frequency dynamics, which can lead to systematically biased results especially in low concentration environments, (ii) sub-diurnal to diurnal scales characterized by a multiplicity of oscillatory components, and (iii) multi-day variations being the essential carrier of information on possible long-range dependence.

A second main finding of this work is that established techniques for time-scale decomposition of observational time series are not necessarily appropriate for clearly distinguishing the effects of the aforementioned three time-scale ranges, since the latter mutually overlap and interact (as far as cyclic components are concerned) with each other in some potentially nonlinear way. Among the available methods, we speculate that empirical mode decomposition [41] might cope somewhat better with this situation than the singular spectrum analysis used in this study, however, on the cost of probably less stable modes estimated by the underlying algorithm. We therefore emphasize that new methodological developments are required to better separate different mutually entangled components contained in radon time series.

Finally, it should be noted that the complex features displayed by radon time series are not unique to this type of record, but can also be found in many other geophysical data sets. Consequently, we believe that the cautionary notes raised in this work and supported by our exemplary analysis apply to many other studies in different fields of Earth sciences as well. To this end, we outline corresponding in-depth investigations of some further examples as a subject of our future research.

\section{Acknowledgements}

This work has been financially supported by the German Federal Ministry for Science and Education via the BMBF Young Investigator's Group CoSy-CC ${ }^{2}$ (grant no. 01LN1306A), the joint Greek-German project "Transdisciplinary assessment of dynamical complexity in magnetosphere and climate: A unified description of the nonlinear dynamics across extreme events" funded by IKY and DAAD (DAAD project no. 56575515, 57161493), and the RADON project (PTDC/CTE-GIX/110325/2009), funded by FCT. S.M. Barbosa acknowledges additional support of the FCT (contract under program IF2013). The authors are grateful to Gideon Steinitz, Heiko Woith, Candelara Martin-Luis and Francisco Lopes for fruitful discussions before, during and after the GeoRadon'14 workshop in Lisbon (Portugal) on 30-31 October 2014, which greatly helped to identify some of the contemporary key problems for radon time series analysis, as well as three anonymous referees for helpful comments on an earlier version of this manuscript.

\section{References}

1. W.W. Nazaroff, Rev. Geophys. 30, (1992) 137-160

2. M. Schubert, L. Brueggemann, K. Knoeller, M. Schirmer, Water Res. Res. 47, (2001) W03512

3. T.-L. Teng, J. Geophys. Res. 85, (1980) 3089-3099

4. E. Hauksson, J.G. Goddard, J. Geophys. Res 86, (1981) 7037-7054

5. R.D. Cicerone, J.E. Ebel, J. Britton, Tectonophysics 476, (2009) 371-396

6. L. Sesana, L. Barbieri, U. Facchini, G. Marcazzan, Radiat. Prot. Dosim. 78, (1998) 65-72

7. World Health Organization. WHO Handbook on Indoor Radon - A Public Health Perspective. (WHO Press, Geneva 2009) 
8. L.J.P.F. Neves, S.M. Barbosa, A.J.S.C. Pereira, J. Environ. Radioactiv. 100, (2009) 896-904

9. S.M. Barbosa, H. Zafrir, U. Malik, O. Piatibratova, Geophys. J. Int. 182, (2010) 829-842

10. H. Woith, S. Barbosa, C. Gajewski, G. Steinitz, O. Piatibratova, U. Malik, J. Zschau, Geochem. J. 45, (2011) 473-482

11. S.M. Barbosa, G. Steinitz, O. Piatibratova, M.E. Silva, P. Lago, Geophys. Res. Lett. 34, (2007) L15309

12. N. Florea, O.G. Duliu, J. Environ. Radioactiv. 104, (2012) 14-23

13. K.Z. Szabó, G. Jordan, Á. Horváth, C. Szabó., J. Environ. Radioactiv. 124, (2013) 74-83

14. W.W. Nazaroff, A.V. Nero, editors, (Wiley 1988)

15. A.V. Nero, A.J. Gadgil, W.W. Nazaroff, K. L. Revzan, Technical Report DOE/ER0480P (Department of Energy, Washington DC 1990)

16. B.J. Smith, R.W. Field, Environmetrics 124, (2007) 481-497

17. G. Cinelli, F. Tondeur, J. Environ. Radioactiv. 143, (2015) 100-109

18. R. K. Churchill, Radon potential in the Lake Tahoe Area, California. Special Report 211 (California Geological Survey 2009)

19. J. Beran. Statistics for Long-Memory Processes. (CRC Press, Boca Raton 1994)

20. A. Witt, B.D. Malamud, Surv. Geophys. 34, (2013) 541-651

21. H.E. Hurst, T. Amer. Soc. Civil Engineers 116, (1951) 770-799

22. M.S. Taqqu, V. Teverovsky, W. Willinger, Fractals 3, (1995) 785-798

23. M.S. Taqqu, V. Teverovsky, A Practical Guide to Heavy Tails (Birkhäuser 1998) p. 177-217

24. J.-M. Bardet, G. Lang, G. Oppenheim, A. Philippe, M.S. Taqqu, Generators of longrange dependent processes: a survey (Birkhäuser 2003) p. 579-623

25. C. Ciancia, U. Facchini, E. Giroletti, S. Rovera, La Radiologia Medica 83, (1992) 282-286

26. E. Giroletti, In Proceedings of the Second Workshop on Radon Monitoring in Radioprotection, Environmental and/or Earth Sciences (World Scientific, Singapore 1993) p. 197-212

27. J. Bejar, U. Facchini, E. Giroletti, S. Magnoni, J. Environ. Radioactiv. 28, (1995) 73-89

28. V. Cuculeanu, A. Lupu, E. Sütö, Environ. Int. 22, (1996) 171-179

29. G. Pausch, P. Bossew, W. Hofmann, in Radon in the Living Environment, Athens, 1999, p. $37-51$

30. J. Planinić, B. Vuković, V. Radolić, J. Environ. Radioactiv. 75, (2004) 35-45

31. V. Radolić, B. Vuković, D. Stanić, J. Planinić, Fizika A 14, (2005) 195-206

32. N.K. Das, H. Chauduri, R.K. Bhandari, D. Ghose, P. Sen, B. Sinha, in Modelling Critical and Catastrophic Phenomena in Geoscience, volume 705 of Lecture Notes in Physics edited by P. Bhattacharyya, B.K. Chakrabarti (Springer, Berlin Heidelberg 2006) p. 481-490

33. N. K. Das, P. Sen, R. K. Bhandari, B. Sinha, Appl. Radiat.Isotopes 67, (2009) 313-318

34. H. Hu, K. Tan, C. Li, J. Lv, D. Liu, Journal of Nanhua University (Science and Technology) 24, (2010) 5-10

35. D. Nikolopoulos, E. Petraki, A. Marousaki, S.M. Potirakis, G. Koulouras, C. Nomicos, D. Panagiotaras, J. Stonham, A. Louizi, J. Environ. Monitor. 14, (2012) 564-578

36. E. Petraki, D. Nikolopoulos, A. Fotopoulos, D. Panagiotaras, G. Koulouras, A. Zisos, C. Nomicos, A. Louizi, J. Stonham, Appl. Radiat. Isotopes 72, (2013) 39-53

37. E. Petraki, D. Nikolopoulos, A. Fotopoulos, D. Panagiotaras, C. Nomicos, P. Yannakopoulos, S. Kottou, A. Zisos, A. Louizi, J. Stonham, Analytical Methods 5, (2013) 4010-4020

38. D. Nikolopoulos, E. Petraki, E. Vogiannis, Y. Chaldeos, P. Yannakopoulos, S. Kottou, C. Nomicos, J. Stonham, J. Radioanal. Nucl. Chem. 299, (2014) 203-219

39. D. Ghosh, A. Deb, S. Dutta, R. Sengupta, S. Samanta, Fractals 20, (2012) 33-39

40. C. Barman, H. Chaudhuri, D. Ghose, A. Deb, B. Sinha, J. Earthquake Science, in press.

41. S. Baykut, T. Akgül, S. İnan, C. Seyis, Radiat. Meas. 45, (2010) 872-879

42. R. G. M. Crockett, F. Perrier, P. Richon, Nat. Hazards Earth Syst. Sci. 10, (2010) 559-564

43. R. G. M. Crockett, G. K. Gillmore, Nat. Hazards Earth Syst. Sci. 10, (2010) 1079-1084 
44. J. Haslett, A.E. Raftery, Appl. Stat. - J. Roy. St. C 38, (1989) 1-50

45. G. Mabille, S. Nicolay, Eur. Phys. J. Special Topics 174, (2009) 135-145

46. S. Nicolay, G. Mabille, X. Fettweis, M. Erpicum, Clim. Dynam. 33, (2009) 1117-1129

47. S. Nicolay, G. Mabille, X. Fettweis, M. Erpicum, Nonlinear Proc. Geophys. 17, (2010) 269-272

48. M. Ghil, M. R. Allen, M. D. Dettinger, K. Ide, D. Kondrashov, M. E. Mann, A. W. Robertson, A. Saunders, Y. Tian, F. Varadi, P. Yiou, Rev. Geophys. 40, (2002) 3

49. R.V. Donner, in Fractal Analysis and Chaos in Geosciences edited by S.-A. Ouadfeul (InTech, Rijeka 2012) p. 1-28

50. Y. Zou, R. V. Donner, J. Kurths, Phys. Rev. E, in press.

51. T. Gneiting, M. Schlather, SIAM Review 46, (2004) 269-282

52. S. Shoupeng, Q. Peiwen, Russ. J. Nondestruct. Testing 43, (2007) 270-280

53. T. Higuchi, Physica D 31, (1988) 277-283

54. G. E. Polychronaki, P. Y. Ktonas, S. Gatzonis, A. Siatouni, P. A. Asvestas, H. Tsekou, D. Sakas, K. S. Nikita, J. Neural Engineering 7, (2010) 046007

55. C.-K. Peng, S.V. Buldyrev, S. Havlin, M. Simons, H.E. Stanley, A.L. Golderberger, Phys. Rev. E 49, (1994) 1685-1689

56. J.W. Kantelhardt, E. Koscielny-Bunde, H.H.A. Rego, S. Havlin, A. Bunde, Physica A 295, (2001) 441

57. K. Hu, P.C. Ivanov, Z. Chen, P. Carpena, H.E. Stanley, Phys. Rev. E 64, (2001) 011114

58. Z. Chen, P.C. Ivanov, K. Hu, H.E. Stanley, Phys. Rev. E 65, (2002) 041107

59. R. V. Donner, S. M. Barbosa, Nonlinear Time Series Analysis in the Geosciences: Applications in Climatology, Geodynamics and Solar-Terrestrial Physics (Springer, Berlin Heidelberg 2008)

60. D. Kondrashov, M. Ghil, Nonlinear Proc. Geophys. 13, (2006) 151-159

61. J. von Buttlar, J. Zscheischler, M. D. Mahecha, Nonlinear Proc. Geophys. 21, (2014) 203-215

62. U. Frisch, Turbulence (Cambridge University Press, Cambridge 1995)

63. K. Bube, C. Rodrigues Neto, R. Donner, U. Schwarz, U. Feudel, J. Phys. D 39, (2006) 1405

64. G. Steinitz, O. Piatibratova, P. Kotlarsky, J. Environ. Radioactiv. 134, (2014) 128-135

65. G. Steinitz, O. Piatibratova, U. Malik, Eur. Phys. J. Special Topics, this issue, (2015)

66. G. Steinitz, M.C. Martin-Luis, O. Piatibratova, Eur. Phys. J. Special Topics, this issue, (2015)

67. M.C. Martin-Luis, G. Steinitz, V. Soler, M.L. Quesada, R. Casillas, Eur. Phys. J. Special Topics, this issue, (2015)

68. J.A.O. Matos, S.M.A. Gama, H.J. Ruskin, A. Al Sharkasi, M. Crane, Physica A 387, (2008) 3910-3915 2008.

69. S. Galmarini, Atmospheric Chemistry and Physics 6, (2006) 2865-2886

70. G. Steinitz, O. Piatibratova, Solid Earth 1, (2010) 99-109 